\title{
Theoretical Analysis of Gas Flow in Shaft with Layered Burdens*
}

\author{
By Mamoru KUWABARA** and Iwao MUCHI**
}

\section{Synopsis}

To clarify the characteristics of gas flow in the shaft of blast furnace having layered ore and coke burdens, the equations of continuity and motion were numerically solved for the isothermal flow of gas through the two-dimensional packed bed.

Solutions are presented both for the case with an average resistance of the adjacent layeres at each radial position and for the case where each resistance of the adjacent layers changes discretely from layer to layer. The flow fields are shown in terms of the patterns of stream-line, mass velocity, and pressure in the bed.

The effects of mass flow rate of gas, compressibility of fuid, shape of bed, and uneven distribution of permeability on the flow fields are discussed on the basis of the computed results for the flow of gas fed into the bed at isobaric conditions. Furthermore, nonuniform flow of gas is related to the charging conditions through an approximate solution.

\section{Introduction}

The radial distributions of the temperature and the composition of gas in the blast furnace may be caused by the uneven flow of gas through the burdens. Accordingly, in the practical operations it is important to clarify the relationship between the characteristic features of gas flow and the operating conditions.

However, because of the complicated behaviors of gas flow in the shaft of operating furnace, the theoretical investigations have not been commenced until recently. For instance, even in the isothermal field without the source from which gas is imagined to flow out the gas dynamics may be affected by the various factors as follows: (1) The furnace geometry, (2) The packed structure of the burdens, and (3) The threedimensional flow of gas fed from the tuyere.

In the majority of the research works presented hitherto, the shape of the furnace has been assumed simply as a rectangle or a cylinder. In connection with (2) mentioned above, Polthier ${ }^{1)}$ inferred that the nonuniform flow of gas through the shaft might result from the differences in the bed height caused by the inclined top surface of the bed. As for (3), Radestock and Jeschar ${ }^{2)}$ presented the numerical analysis on the flow of the compressible gas through the cylindrical region around raceway. In addition, Shvydkii, et al..$^{3)}$ analyzed the incompressible and irrotational flow through the rectangular bed with uniformly packed coke.

On the other hand, there exist a few theoretical studies in which spatially nonuniform resistance to flow is taken into account. Radestock and Jeschar ${ }^{2}$ ) dealt with the system having both the layered burdens with the same voidage and the deadman with the large resistance to flow. Stanek and Szekely ${ }^{4}$ carried out the numerical analysis on the incompressible flow through the rectangular packed bed having the simple nonuniform structure. They, however, adopted the imaginary boundary condition such that the inlet flow rate of gas may be uniform independent of the laterally distributed resistance. Araki, et al. ${ }^{5}$ presented the approximate analysis on the flow of incompressible fluid through the parallel packed beds consisting of two kinds of solid particles. In their analysis, the condition of gas flow was confined to the laminar region.

In the shaft of blast furnace with layered ore and coke burdens, the different apparent angles of repose of both layers naturally cause the nonuniformity of the resistance to flow. Previously, present authors ${ }^{6)}$ gave the expressions to describe such inequality in the resistance longitudinally meaned at each radial position. Furthermore, the authors ${ }^{6)}$ presented a mathematical model to predict the radial distribution of gas flow by the use of the operating conditions. The model was developed for the case of a cylindrical furnace without a deadman, and an approximate solution for such system was derived from the equations of continuity and motion.

In the present paper, to clarify the effects of the compressibility of fluid, the inclined top surface of bed, the deadman, and the softening layer on the behavior of gas flow, the equations of continuity and motion have been solved numerically for the various shapes of the packed bed with the layered burdens. The result is shown for each case of the beds having the continuously distributed resistance and the discretely distributed resistance. In order to confirm the availability of the approximate analysis previously presented, ${ }^{6}$ ) the exact solution obtained by the numerical computation is compared with the approximate solution.

\section{Flow Equations and Numerical Method}

\section{Flow Equations}

To simulate the shaft of blast furnace, a two-dimensional packed bed with layered burdens is considered as shown in Fig. 1. Moreover, let us consider the isothermal and compressible fluid ascending through the bed. It is readily expected that the spatially distributed resistance to flow may cause the spatialy nonuniform flow of gas through the furnace. That is the

* Originally published in Tetsu-to-Hagané, 62 (1976), 463, in Japanese. English version received July $17,1976$.

** Department of Iron and Steel Engineering, Nagoya University, Furo-cho, Chikusa-ku, Nagoya 464. 
problem to be analyzed as the following.

In general, the equation of continuity in the flow field without a source, from which the fluid is imagined to flow out, can be written as follows:

$$
\partial(\varepsilon, \rho) / \partial t=-\operatorname{div}(\varepsilon \rho \vec{v})
$$

Under the steady state condition, the left-hand side of Eq. (1) vanishes in either case of the compressible fluid or the incompressible one. Then,

$$
\operatorname{div}(\varepsilon \rho \vec{v})=0
$$

Regardless of the compressibility of fluid, the differential version of Ergun's equation ${ }^{7}$ can be approximately substituted for the equation of motion. Accordingly, the pressure drops to be caused in the $r$ - and $l$-directions may be described by:

$$
\begin{aligned}
& \partial P / \partial r=-\left(f_{1}+f_{2}\left|G_{r}\right|\right) G_{r} \\
& \partial P / \partial l=-\left(f_{1}+f_{2}\left|G_{l}\right|\right) G_{l}
\end{aligned}
$$

where,

$$
\begin{aligned}
& f_{1} \equiv 150(1-\varepsilon)^{2} \mu / g_{c} \rho\left(\phi d_{p}\right)^{2} \varepsilon^{3} \\
& f_{2} \equiv 1.75(1-\varepsilon) / g_{c} \rho\left(\phi d_{p}\right) \varepsilon^{3}
\end{aligned}
$$

Here, the stream function $(\Psi)$ is defined as follows:

$$
u=(\partial W / \partial l) / \varepsilon_{\rho} \rho, \quad v=-(\partial W / \partial r) / \varepsilon_{\rho} O
$$

Namely,

$$
G_{r}=\partial \psi / \partial l, \quad G_{l}=-\partial \Psi / \partial r
$$

It goes without saying that the equation of continuity can be naturally satisfied by introducing $\Psi$. Upon eliminating $P$ from Eq. (3) and writing the resultant equation in terms of $\Psi$ as proposed by Stanek and Szekely, ${ }^{4)}$ the fundamental equation of flow can be obtained as:

$$
\begin{gathered}
\frac{\partial^{2} \Psi}{\partial r^{2}}\left(f_{1}+2 f_{2}\left|\frac{\partial \Psi}{\partial r}\right|\right)+\frac{\partial^{2} \Psi}{\partial l^{2}}\left(f_{1}+2 f_{2}\left|\begin{array}{c}
\partial \Psi \\
\partial l
\end{array}\right|\right) \\
+\frac{\partial \Psi}{\partial r}\left(\frac{\partial f_{1}}{\partial r}+\frac{\partial f_{2}}{\partial r}\left|\frac{\partial \Psi}{\partial r}\right|\right) \\
+\frac{\partial \Psi}{\partial l}\left(\frac{\partial f_{1}}{\partial l}+\frac{\partial f_{2}}{\partial l}\left|\frac{\partial \Psi}{\partial l}\right|\right)=0 \quad \ldots \ldots . . . . .
\end{gathered}
$$

Ultimately, the stream function field can be determined by solving Eq. (6), and also the fields of the mass velocity and the pressure can be evaluated from Eqs. (5)' and (3), respectively.

\section{Boundary Conditions}

The symmetrical condition on the center axis is readilly expressed as:

$$
G_{r}=0
$$

From the condition expressing the wall to be impervious to flow,

$$
G_{r} \sin \theta_{2}+G_{l} \cos \theta_{2}=0
$$

where, $\theta_{2}$ denotes the angle between the bottom and wall planes, and its value is taken to be $83^{\circ}$ for an imaginary shaft and $90^{\circ}$ for a rectangular bed.

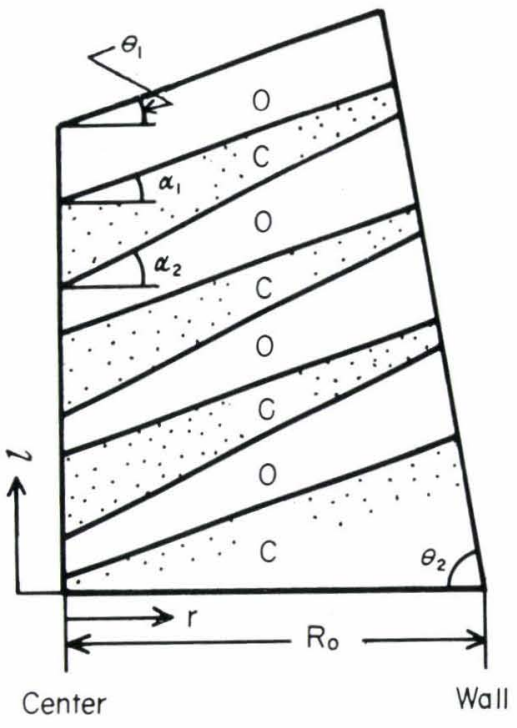

Fig. 1. Schematic view of layered burdens

It would be really probable that no pressure distribution may exist along the top surface of the bed, hence the condition at the outlet can be given as follows:

$$
G_{r} \cos \theta_{1}+G_{l} \sin \theta_{1}=0, \quad P=P_{\text {out }}
$$

where, $\theta_{1}$ designates the apparent angle of repose of the particle layer at the top of the bed.

At the bottom of the bed, it may be reasonable to impose the isobaric condition on the gas to be fed into the bed. Accordingly $G_{l}$ becomes a function of the lateral position due to the lateral distribution of the hydraulic resistance. Namely,

$$
G_{l}=G_{l}(r), \quad P=P_{\text {in }}
$$

When these boundary conditions are expressed in terms of $\Psi$, Eqs. (7) and (8) imply that there does not exist the changes of $\Psi$ along the center axis and the side wall, respectively. Rewriting Eq. (9) into the finite-difference representation, we have:

$$
\Psi_{i, j+1}=\Psi_{i, j}+\tan \theta_{1} \cdot(\Delta l / \Delta r)\left(\Psi_{i+1, j}-\Psi_{i, j}\right) \ldots
$$

where, the space point $(i \Delta r, i \Delta l)$, also called the lattice point $(i, j)$, is surrounded by the neighboring lattice points.

From Eq. (11), $\Psi_{i, j+1}$ at the top of the bed can be determined by using the stream functions at two lattice points $(i, j)$ and $(i+1, j)$.

The value of $G_{l}(r)$ in Eq. (10), accordingly $\Psi(r)$ at the inlet, was determined according to the following procedures. Firstly, the initial value of $\Psi(r)$ is arbitrarily given according to the quadratic equation shown by:

$$
\Psi(r)=a r^{2}+b r+c
$$

where, $a, b$ and $c$ are the constants.

Secondly, Eq. (6) is solved numerically for $\Psi$ considering the boundary conditions mentioned above. We can now proceed to obtain the streamlines as well 
as the values of $G_{r} G_{l}, \partial P / \partial r$ and $\partial P / \partial l$ at all space points.

Thirdly, the pressure drops along each streamline are integrated starting from the same inlet pressure, $P_{\text {in }}$. Concerning these overall pressure drops across the bed, a standard deviation $(s)$ is calculated.

Finally, the same procedure mentioned above is iterated modifying the values of the coefficients in Eq. (12) until we get a minimum standard deviation.

For the sake of convenience, the value of $c$ in Eq. (12) may be assumed to be equal to zero, since it is of no effect on the calculated results of the streamline and the mass velocity. Moreover, upon designating the mean mass velocity at the inlet $\left(G_{0}\right)$ as given by Eq. (13), the functional relation between $a$ and $b$ can be found as follows:

$$
G_{0}=\int_{0}^{R_{0}} G_{l}(r) d r / R_{0}=-a R_{0}-b
$$

After all, $\Psi(r)$ is expressed by the followings:

$$
\Psi(r)=a r^{2}-\left(a R_{0}+G_{0}\right) r
$$

Consequently, the problem to determine $\Psi(r)$ at the inlet under the isobaric condition can be solved from the univariant search for determining the optimum value of the coefficient $a$. From the physical constraint, the value of $a$ is restricted within the domain represented by Eq. (15). Namely, a stipulation of $G_{l}(r)>0$ at $0 \leqq r \leqq R_{0}$ together with Eqs. (14) and (5)' yields:

$$
-\left(G_{0} / R_{0}\right)<a<\left(G_{0} / R_{0}\right)
$$

\section{Numerical Solution}

Fundamental flow equation (Eq. (6)) was rewritten into the finite-difference representation and was numerically solved by the method of successive overrelaxation (S.O.R.). Spacially distributed variables in the bed were calculated at all lattice points whose numbers amounted to 4000 to 10000 , according to the objects to be computed. A large number of lattice points were utilized for the case of the inclined top contour of the burdens or the inclined wall, but for the case of the rectangular bed the computed results were not affected even if the lattice points more than 4000 were taken.

To accelerate the convergence, a relaxation parameter $(\omega)$ at every step of the iterations was successively modified so that the convergence factor $(\lambda \equiv$ $\left.\|\delta\|_{m} /\|\delta\|_{m-1}\right)$ may become smaller than the predetermined value $\left(\lambda_{i}\right)$. Where, $\|\delta\|_{m}$ is the Euclidian norm in the $m$-th iteration and is defined in temrs of the residuals of stream function $(\delta)$ at each lattice point.

For a choice of $\lambda_{i}$ in a little greater range than 1.0, a rapid convergence could be obtained. According to the objects to be computed, the optimum value of $\omega$ ranged from 0.80 to 1.90 . The overrelaxation was brought to an end when a maximum discrepancy of $0.005 \%$ in the stream function was reached. The computing time involved was about $1.5 \mathrm{~min}$ on FACOM 230-75 digital computer.

\section{Flow of Incompressible Fluid}

In this section, the analyses on the isothermal flow of gas are described for the case where the change of its density due to the pressure may be negligible. In other words, the results are applicable for the case of $\left(P_{\text {in }} / P_{\text {out }}\right) \cong 1$.

To clarify the effect of the fluid compressibility on the flow pattern, a physical model has been supposed. Numerical values to be used in this model were scaled down on the basis of the geometrical similarity and the Reynolds' one, and the values are given in Table 1. It can be found from Eq. (6) that the characteristic features in the flow field are not affected by the reduced extent of the values because the geometric similarity is held

The value of $G_{0}$ is chosen as 0.1 or $10 \mathrm{~kg} / \mathrm{m}^{2}$ (bed). sec, according as the flow is laminar or turbulent. Calculated results are illustrated for the following two types of the resistence distributions in a bed having the layered burdens; these are:

(1) Continuous distribution of resistance: Such distribution is given by averaging each resistance of the adjacent coke and ore layers at the individual lateral position.

(2) Discrete distribution of resistance: In the actual bed with the inclined layers of burdens there, of course, exists the specially distributed resistance. Such distribution arises from the difference in the resistance between the adjacent layers.

\section{Continuously Distributed Resistance}

According to the same procedures as shown in the previous paper, ${ }^{6}{ }^{6}$ the voidage and the particle diameter in each layer of two dimensional bed are averaged at each lateral position. That is, two adjacent layers of coke and ore which are located from $r$ to $(r+d r)$ are considered as a cell having the averaged voidage. Calculating the voidage of the cell leads to the following linear expression:

$$
\bar{\varepsilon}=A+B r
$$

where,

\begin{tabular}{|c|c|c|c|}
\hline & & Coke & Ore \\
\hline & $(\mathrm{m})$ & 0.003 & 0.001 \\
\hline$\varepsilon$ & $(-)$ & 0.40 & 0.35 \\
\hline$\alpha$ & $(-)$ & $25^{\circ}$ & $30^{\circ}$ \\
\hline$\phi$ & $(-)$ & 1.0 & 1.0 \\
\hline$V$ & $\left(\mathrm{~m}^{3}\right.$ (bed $\left.)\right)$ & $3.2 \times 10^{-4}$ & $3.2 \times 10^{-4}$ \\
\hline$R_{0}$ & $(\mathrm{~m})$ & \multicolumn{2}{|c|}{0.2} \\
\hline & $(\mathrm{m})$ & \multicolumn{2}{|c|}{0.04} \\
\hline & $(\mathrm{kg} / \mathrm{m} \cdot \mathrm{sec})$ & \multicolumn{2}{|c|}{$1.8 \times 10^{-5}$} \\
\hline$\rho$ & $\left(\mathrm{kg} / \mathrm{m}^{3}\right)$ & \multicolumn{2}{|c|}{1.2} \\
\hline$P_{\text {out }}$ & $\left(\mathrm{Kg} / \mathrm{m}^{2}\right)$ & \multicolumn{2}{|c|}{$1.033 \times 10^{4}$} \\
\hline
\end{tabular}

$$
\begin{aligned}
A & \equiv\left(\Delta_{1} \varepsilon_{1}+\Delta_{2} \varepsilon_{2}\right) / \Delta, \quad B \equiv\left(\varepsilon_{2}-\varepsilon_{1}\right) \Lambda / \Delta, \\
\Delta & =\Delta_{1}+\Delta_{2}, \quad \Delta_{1}=V_{1} / R w+R \Lambda / 2, \\
J_{2} & =V_{2} / R w-R \Lambda / 2, \quad \Lambda \equiv \tan \alpha_{2}-\tan \alpha_{1}
\end{aligned}
$$

Table 1. Data for numerical calculation 
Assuming the overall hydraulic diameter of a cell as the harmonic means of both the hydraulic diameters of coke and ore in the cell, the particle diameter averaged longitudinally can be derived as:

$$
\bar{d}_{p}=\Delta(1-\bar{\varepsilon}) /(C+D r) \cdot \bar{\varepsilon}
$$

where,

$$
\begin{aligned}
& C \equiv \Delta_{1}\left(1-\varepsilon_{1}\right) / d_{p 1} \varepsilon_{1}+J_{2}\left(1-\varepsilon_{2}\right) / d_{p 2} \varepsilon_{2} \\
& D \equiv\left\{\left(1-\varepsilon_{2}\right) / d_{p 2} \varepsilon_{2}-\left(1-\varepsilon_{1}\right) / d_{p 1} \varepsilon_{1}\right\} A
\end{aligned}
$$

Figure 2 shows the calculated results of the streamlines and the patterns of $G_{l}$ in a rectangular bed. The perpendicularly distributed streamlines may tell us that for any mass velocity of gas in a rectangular bed, there would not exist any cross-flow and accordingly vertical change in the patterns of $G_{l}$. As is shown from the positions of streamlines or the patterns of $G_{l}$, the more the flow rate decreases, the more the central flow increases. However, for $G_{0}$ above $3 \mathrm{~kg} / \mathrm{m}^{2}$ (bed). $\mathrm{sec}$, insignificant effect of the flow rate of gas on these curves was found.

Figure 3 shows the effects of the inclined top surface on the streamlines and the patterns of $G_{l}$. Near the top of the bed, all streamlines tend to intersect perpendicularly to the outlet plane and to shift the positions of these lines toward the central axis. Consequently, the central flow of gas is promoted. Moreover, in the neighbourhood of the bottom of bed, the streamlines have comparatively large curvatures. However, the dotted lines in Fig. 3, which correspond to a laminar state, intersect almost perpendicularly to the inlet plane. These results mentioned above may suggest that the curved streamlines in the bottom of the bed might be attributable to the effect of the nonlinear resistance which is shown by the second term in the right-hand side of Eq. (3), $f_{2} G \vec{G}$.

The results calculated for the bed having the inclined wall are illustrated in Fig. 4. It is to be noted in Fig. 4 that the mass velocity of gas flowing along the inclined wall tends to increase but this tendency is not so remarkable.

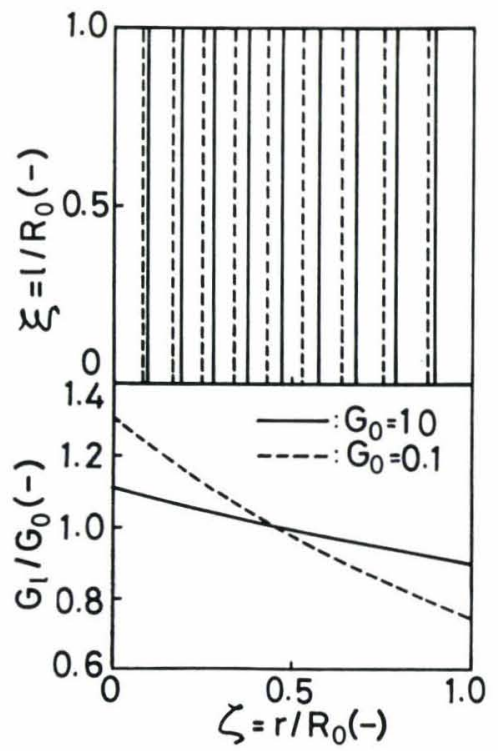

Fig. 2. Streamlines and patterns of $G_{l}$ for bed with the continuously distributed resistance
Figure 5 shows the patterns of the streamline, the isobar, and $G_{l}$ in the case of a bed having the inclination of the top surface and the inclined wall. In the present instance each streamline is considered approximately straight except for the regions near the bottom and the top. Consequently, it can be infered that the gas flow in the intermediate region of the bed is principally governed by the nonuniform distribution of resistance.

\section{Comparison with Approximate Solution}

On the basis of the results described above for a rectangular bed or a bed having a similar profile to the shaft of blast furnace, an approximate solution is derived for the nonuniform flow of gas through the two-dimensional packed bed where no cross-flow exists.

Ergun's equation including the lateral distribution of resistance can be written by:

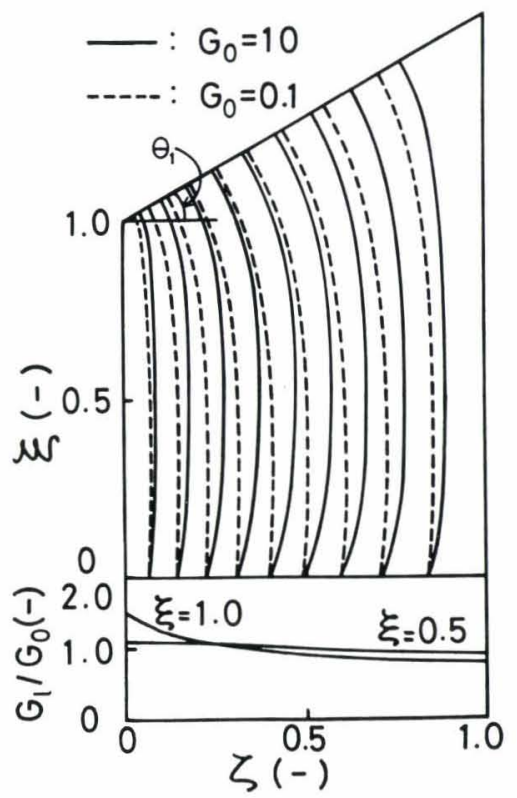

Fig. 3. Streamlines and patterns of $G_{l}$ for bed with the continuously distributed resistance $\left(\theta_{1}=30^{\circ}\right)$

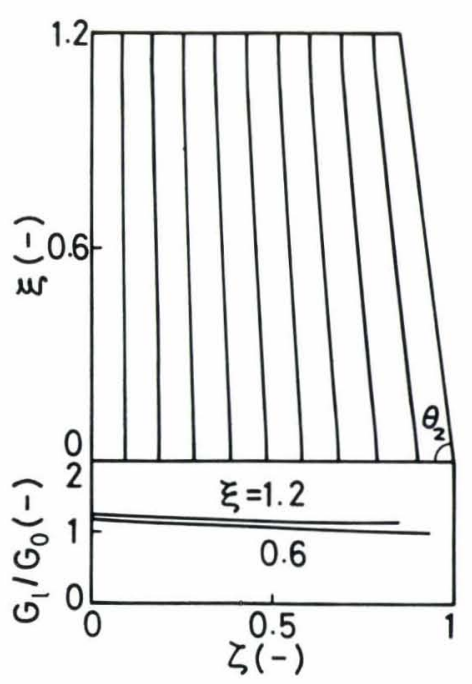

Fig. 4. Streamlines and patterns of $G_{l}$ for bed with the continuously distributed resistance $\left(\theta_{2}=83^{\circ}\right)$ 


$$
\partial P / \partial l=-\bar{\Psi}(r) \cdot(1-\bar{\varepsilon})\left\{G_{l}(r)\right\}^{2} / g_{c} \rho \cdot \bar{\phi}(r) \cdot \bar{d}_{p} \bar{\varepsilon}^{3}
$$

where, $\bar{\Psi}(r)$ is nearly constant at high Reynolds number and $\bar{\phi}(r)$ is also considered to be constant as mentioned in the previous paper. $\left.{ }^{6}\right)$ by :

The condition without cross-flow can be expressed

$$
\partial(\partial P / \partial l) / \partial r=0
$$

Furthermore, the mass flow rate of flowing gas can be written by:

$$
F_{g}=w \int_{0}^{R} G_{l}(r) d r
$$

Upon substituting Eqs. (16) and (18) to $\bar{\varepsilon}$ and $\bar{d}_{p}$ in Eq. (20), and combining Eqs. (21) and (22), an approximate solution for the lateral distribution of the mass velocity of gas can be derived as follows:

$$
G_{l}(r)=F_{g}(A+B r) / w E \sqrt{ } C+D r
$$

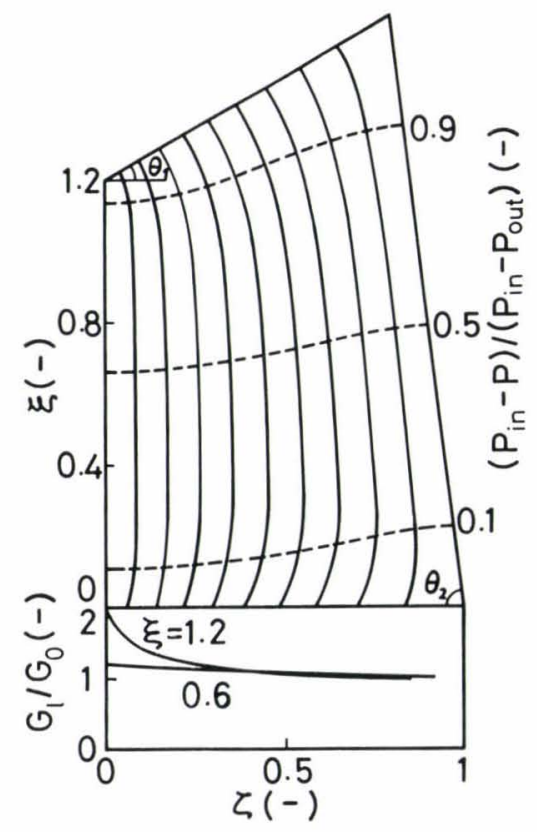

Fig. 5. Streamlines, patterns of $G_{l}$ and isobar for bed with the continuously distributed resistance $\left(\theta_{1}=30^{\circ}, \theta_{2}\right.$ $\left.=83^{\circ}\right)$ where, $E$ is given by Eq. (24) for the case of $\alpha_{1} \neq \alpha_{2}$, and by Eq. (24)' for $\alpha_{1}=\alpha_{2}$.

$$
\begin{aligned}
E \equiv & 2\{3(A D-B C)(\sqrt{ } C+D R-\sqrt{ } C) \\
& +B[(C+D R) \sqrt{ } C+D R-C \sqrt{ } C]\} / 3 D^{2} \\
E \equiv & R A / \sqrt{ } C \ldots \ldots \ldots \ldots \ldots \ldots \ldots \ldots \ldots \ldots \ldots \ldots \ldots \ldots \ldots \ldots \ldots \ldots \ldots \ldots \ldots \ldots \ldots
\end{aligned}
$$

A close agreement was obtained between the numerical solution for the pattern of $G_{l}$ shown in Fig. 2 $(s=0.06 \%)$ and the approximate solution determined from Eq. (23), provided the Reynolds number based on particle diameter at the position of $r=R / 2$ is higher than about 700 . Therefore, the effects of the operating conditions on the patterns of $G_{l}$ at high Reynolds number can be easily predicted from Eq. (23) without a laborious numerical computation. Equation (23) would inform us, for example, that the flow pattern of gas may become uniform under the charging conditions having: the increased ratio of ore/coke, increased coke base, and the decreased difference of apparent angles of repose between the adjacent layers of coke and ore.

\section{Discrete Distribution of Resistance}

The computed results are given on gas flow in the bed, where the resistances in the adjacent layers are discretely distributed due to the layered configuration of burdens.

Figure 6 shows the results obtained on the streamlines, the mass velocity, and the isobar in a rectangular bed. As is obvious from Fig. 6 (a), the ascending gas has a tendency to flow along the path having the smaller resistance and consequently the zigzag streamlines result with passing through the alternate layers. Figure 6(b) shows that the mass velocity of gas changes remarkably in the lateral as well as vartical directions except for the region near to the top and the bottom. Namely, it is seen that there exist a central flow at $\xi=0.2$ and a peripheral flow at $\xi=0.8$, while the uniform rate of flow appears at $\xi=0$ and 1 . Such phenomena indicates the preferential flow of gas through the coke layer. Figure 6 (c) shows the marked lateral distribution of the isobar refracted at the neighbourhood of the interface between the adjacent layers. Such nonuniformity also arises from the large difference in flow resistances between the ad-

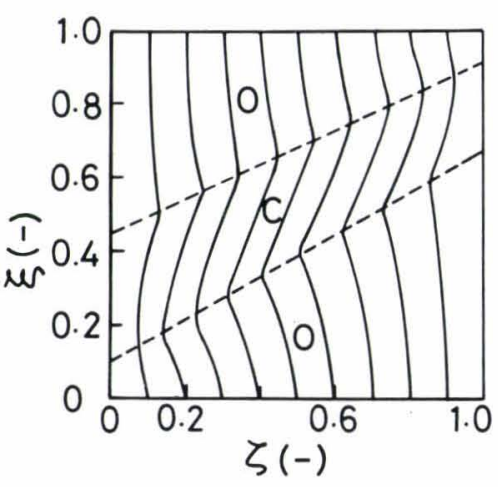

(a) streamlines

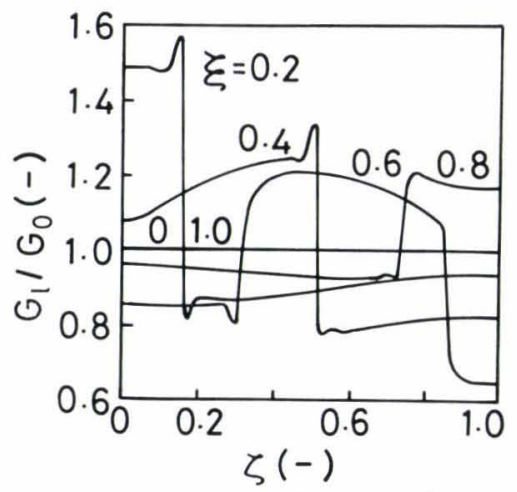

(b) patterns of $G_{1}$

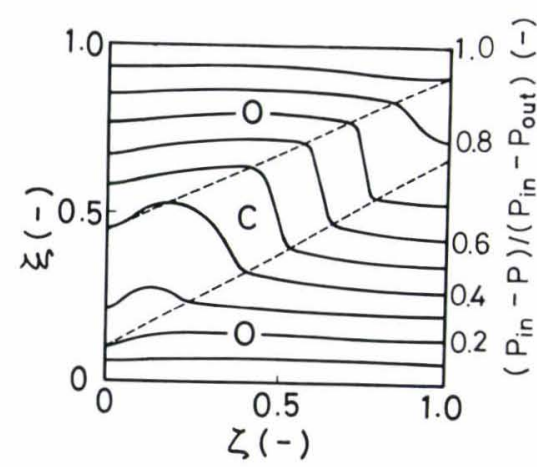

(c) isobars

Fig. 6. Streamlines, patterns of $G_{l}$ and isobar for layered burdens with the discretely distributed resistance 
jacent layers.

Figure 7 shows the zigzag pattern of streamlines and the remarkable change of the mass velocity in the bed having a similar profile to the shaft of a blast furnace. It is interesting to note that the averages of these oscillating values approximately coincide with the results shown in Fig. 5 where the resistance is continuously distributed. An analogous but other calculation indicated that the centralized flow near the inclined top is less when the ore layer locates at the top of the furnace, compared with the case having the coke layer at the top.

Figures 8 to 10 show the patterns of streamline for the rectangular bed having a deadman. In the computations, the angle between the inlet plane and the contour of a deadman of coke is assumed as $\theta_{3}=70^{\circ}$.

Figure 8 shows the pattern of streamlines obtained for the case of the extremely small rate of flow. The intervals between the adjacent streamlines suggest that the flowing gas takes the roundabout way to avoid the ore layer with the larger resistance. Consequently, a large quantity of gas tends to flow through a deadman.

Figure 9 shows the pattern of streamlines for the case of the comparatively large rate of flow. The central flow also increases due to the tendency to keep away from the ore layer. However, this tendency is remarkably suppressed in comparison with the results illustrated in Fig. 8. This may suggest that the ascending gas becomes easy to penetrate into the ore layer with the increased mass flow rate of gas, accordingly with the increased inertial force given by the second term of Ergun equation.

Recently, Kanbara, et al. ${ }^{8)}$ detected the annular softening layers of ore existing around a deadman on the basis of the survey from the dissected furnace. From the model experiment, Ono, et al. ${ }^{9)}$ reported that the hydraulic resistance of a softening layer was remarkably large.

In the present study the softening layers, which presumably have the voidage of 0.2 and the same particle diameter as the ore particle, were arranged

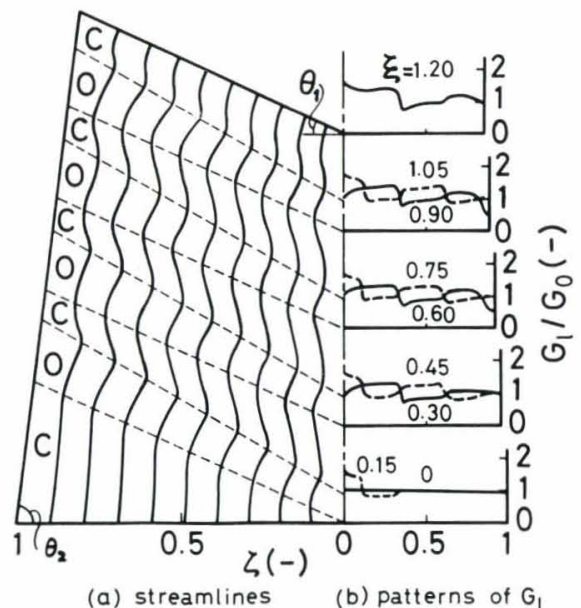

Fig. 7. Streamlines and patterns of $G_{l}$ for layered burdens with the discretely distributed resistance $\left(\theta_{1}=30^{\circ}\right.$, $\theta_{2}=83^{\circ}$ )

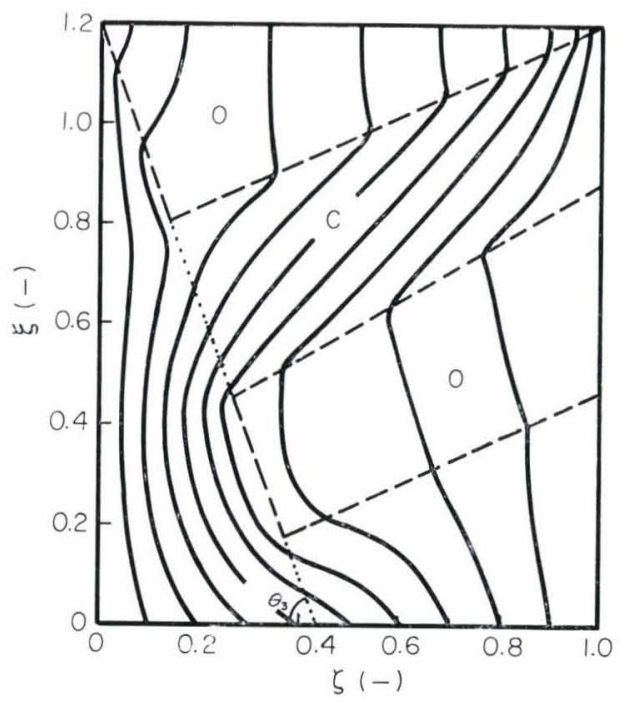

Fig. 8. Streamlines in rectangular bed with a deadman at low mass velocity of gas $\left(G_{0}=0.1 \mathrm{~kg} / \mathrm{m}^{2}\right.$ (bed) $\cdot \mathrm{sec}$, $\left.\theta_{3}=70^{\circ}\right)$

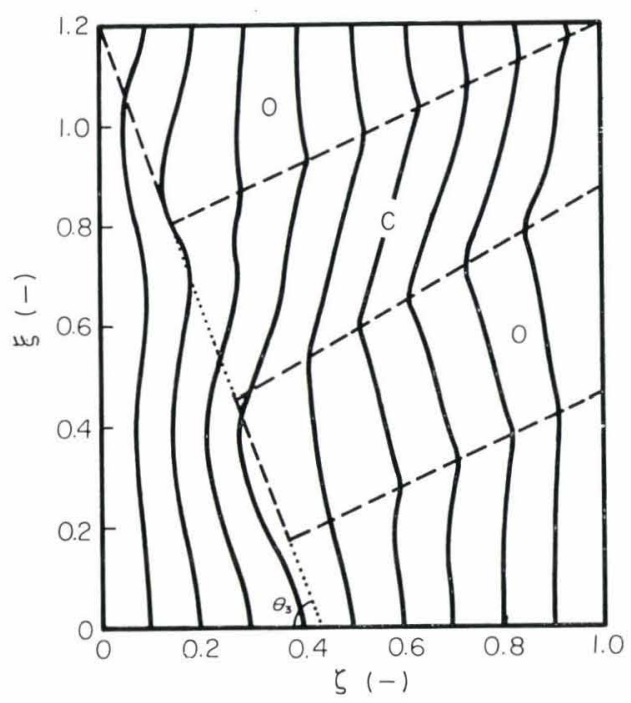

Fig. 9. Streamlines in rectangular bed with a deadman at high mass velocity of gas $\left(G_{0}=10 \mathrm{~kg} / \mathrm{m}^{2}\right.$ (bed) $\left.\cdot \mathrm{sec}\right)$

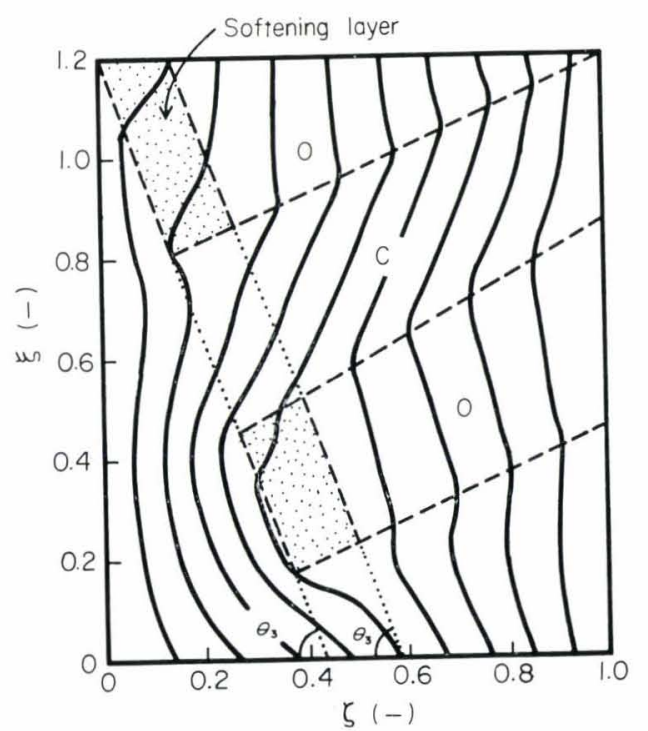

Fig. 10. Streamlines in rectangular bed with a deadman and the softening layers at $G_{0}=10 \mathrm{~kg} / \mathrm{m}^{2}$ (bed) $\cdot \mathrm{sec}$ 
around a deadman in the rectangular bed. It is to be noted in Fig. 10 that the streamlines tend to bunch in the deadman despite of a large flow rate of gas. In consequence, the central flow is increased more than one in Fig. 9. The softening layers, therefore, would be responsible for the centralized flow through a deadman. However, the effects of the softening layers on the fluid flow may be restricted to the region comparatively near to the layers.

\section{Flow of Compressible Fluid}

The change of gas density has to be considered when the value of $\left(P_{\mathrm{in}} / P_{\text {out }}\right)$ largely exceeds 1.0 due to the large flow rate or the large resistance of bed. This section is devoted to the numerical analysis on the isothermal flow of the compressible gas through the bed having nonuniform permeability.

\section{Procedure of Numerical Analysis}

Equation (6) is applicable both for the compressible and incompressible flows. Considering the compressibility, the gas density involved in the drag coefficients $\left(f_{1}\right.$ and $\left.f_{2}\right)$ has to be given as a function of the position. The numerical analysis is conducted as the following procedure.

Firstly, the flow field is computed by neglecting the compressibility, and from its field, the pressure field is determined. Secondly, the spatial distribution of gas density is evaluated with the aid of the equation of state. By using its distribution, the spatially variable values of $f_{1}$ and $f_{2}$ are determined. Thirdly, the computation on the pressure field is performed again by taking account of $f_{1}$ and $f_{2}$. Finally, the same procedure mentioned above is repeated until we get the invariable density of gas on every lattice point. The final flow patterns give the flow field for - the case including the compressibility.

Figure 11 shows the flow chart for the procedure mentioned above. To determine the distribution of pressure in the bed, $P(r, l)$, the isobaric inlet pressure $\left(P_{\mathrm{in}}\right)$ is given by:

$$
P_{\text {in }}=P_{\text {out }}+(\Delta P)_{\text {bed }}
$$

where, $P_{\text {out }}$ denotes the outlet pressure arbitrarily predetermined and $(\Delta P)_{\text {bed }}$ the total pressure drop to be caused along each streamline. Then, $P(r, l)$ can be determined as follows:

$$
P(r, l)=P_{\text {in }}+\int_{0}^{l}(\partial P / \partial l)_{r} d l
$$

where, $(\partial P / \partial l)_{r}$ denotes the pressure gradients at each lateral position.

Furthermore, the density $\rho(r, l)$ can be evaluated from:

$$
o(r, l)=\left(M / R_{g} T\right) \cdot P(r, l)
$$

\section{Computed Results}

The results calculated by including the compressibility are described for each case of (a) the rectangular bed with the continuously distributed resistance and (b) the rectangular bed having a deadman and the discretely distributed resistance.

The computed results show the very small effects of the compressibility on th pattern of streamlines in both cases. Namely, the results obtained for each case of (a) and (b) were practically similar to the results shown in Fig. 2 and Fig. 9, respectively.

As far as case of (a) is concerned, no lateral distributions of pressure of gas were found independent of its compressibility. However, the longitudinal variation in the pressure of a compressible fluid differs appreciably from that of an incompressible one. Figure 12 shows that the latter is straight while the former becomes curvilinear owing to the gradual decrease in the density of the ascending gas.

Figure 13 shows the isobars obtained for the case of (b). It is worth noting that the isobars at the compressible flow are shifted upwardly, but the patterns of isobar distribution in both cases are resemble each other.

Consequently, the numerical analysis on the incompressible flow would give the valid informations concerning the field of stream function and accordingly the fields of streamlines and mass velocity, even if there may exist the discretely distributed resistance in the system. However, in the determination of the pressure field it may be necessary to take account of the compressibility of the fluid.

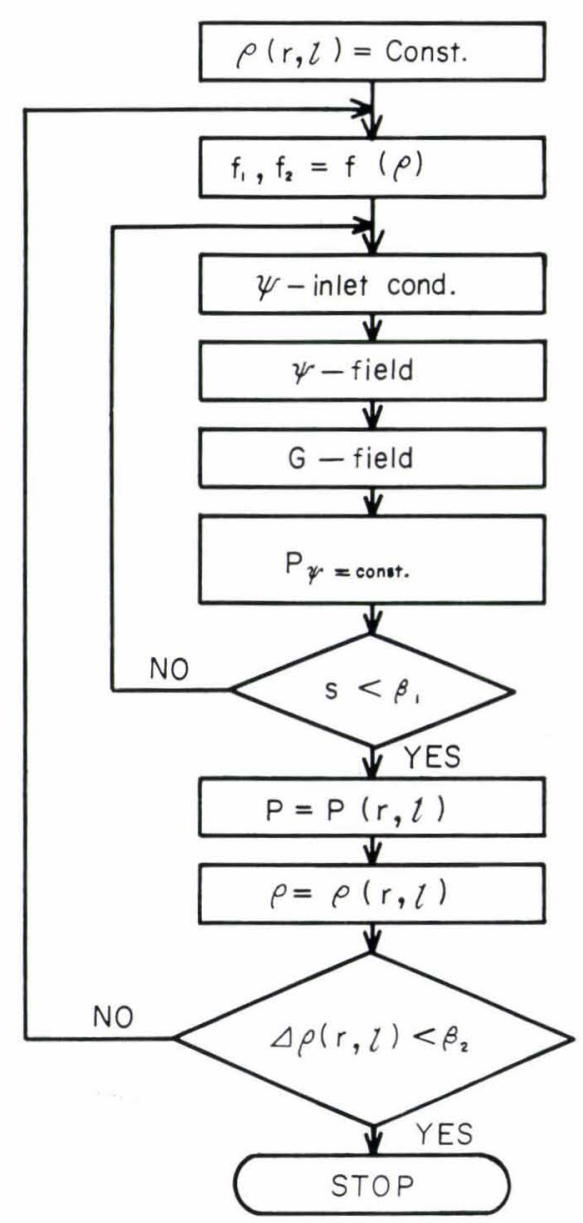

Fig. 11. Flow chart for compressible flow 


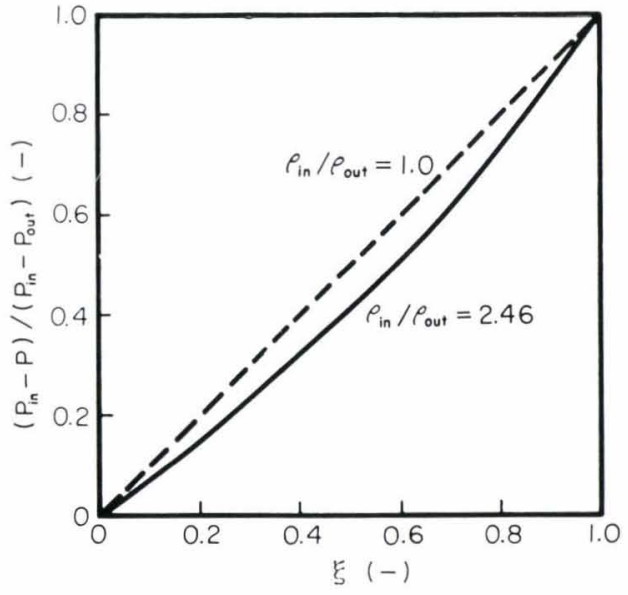

Fig. 12. Longitudinal distribution of gas pressure in rectangular bed with the continuously distributed resistance at $G_{0}=10 \mathrm{~kg} / \mathrm{m}^{2}$ (bed) $\cdot$ sec

\section{Conclusions}

The numerical analysis and the approximate one have been developed to clarify the characteristics of gas flow through the shaft of blast furnace having layered coke and ore burdens. In the numerical analysis, the compressibility of the fluids has also been taken into account.

In the first place, the fluid flow through the bed has been numerically analyzed for the case where longitudinally averaged resistance of the adjacent layers distributes continuously in the lateral direction. As the results, some quantitative informations have been obtained concerning the effects of the radial distributions of resistance to flow, the inclined top contour of burdens, and the inclined wall of bed on the nonuniform fluid flow. From the comparison of the results between numerical analysis and approximateone, it has been found that the effects of the operating conditions on the velocity profiles of gas can be easily predicted by using a simlle theoretical equation derived from the approximate analysis.

In the second place, the numerical analysis on the fluid flow has been carried out for the case where each resistance of the adjacent layers changes discretely from layer to layer. Computed results gave the spatially nonuniform flow as shown by the zigzag pattern of the streamlines and the refracted distributions of the mass velocity and the pressure of the fluid. Such characteristic flow of the fluid may result from the preferencial flow through the coke layers having the smaller resistance.

Furthermore, from the numerical analysis including the compressibility of fluid it has been found that the distributions of the streamline and the mass velocity can be readily determined by assuming the fluid incompressible. However, the general nature of the pressure distribution has to be estimated by taking account of the compressibility of the fluid.

\section{Nomenclature}

$d_{p}$ : diameter of solid particle $(\mathrm{m})$

$F_{g}$ : mass flow rate of gas $(\mathrm{kg} / \mathrm{hr})$

$g_{c}$ : conversion factor $\left(\mathrm{kg} \cdot \mathrm{m} / \mathrm{Kg} \cdot \mathrm{hr}^{2}\right)$

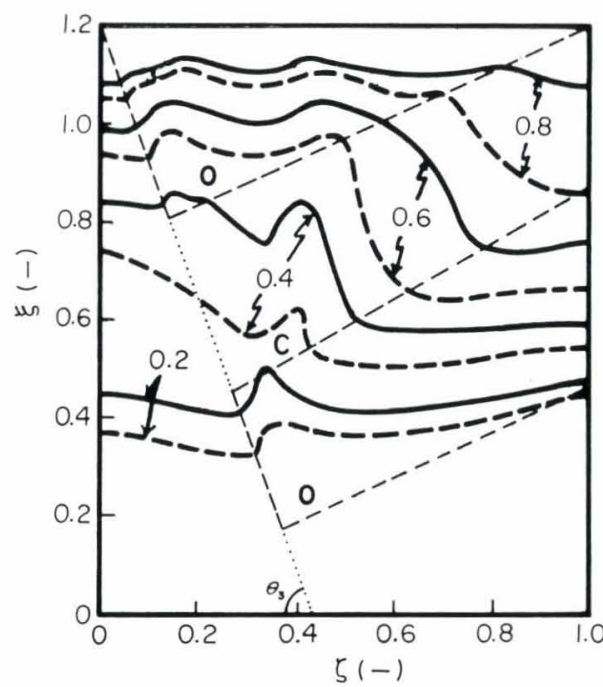

$$
\begin{aligned}
& -\quad \rho_{\text {in }} / \rho_{\text {out }}=2.36, \\
& =-\rho_{\text {in }} / \rho_{\text {out }}=1.0, \\
& \text { parameter: } \quad\left(P_{\text {in }}-P\right) /\left(P_{\text {in }}-P_{\text {out }}\right)
\end{aligned}
$$

Fig. 13. Patterns of isobars in rectangular bed with a dead$\operatorname{man}$ at $G_{0}=10 \mathrm{~kg} / \mathrm{m}^{2}$ (bed) $\cdot \mathrm{sec}$

$G$ : mass velocity of gas $\left(\mathrm{kg} / \mathrm{m}^{2}\right.$ (bed) $\left.\cdot \mathrm{hr}\right)$

$G_{l}, G_{r}$ : longitudinal and radial components of $G$, respectively $\left(\mathrm{kg} / \mathrm{m}^{2}\right.$ (bed) $\left.\cdot \mathrm{hr}\right)$

$l$ : distance from bottom of bed $(\mathrm{m})$

$M$ : molecular mass of gas $(\mathrm{kg} / \mathrm{kgmol})$

$P:$ pressure of gas $\left(\mathrm{kg} / \mathrm{m}^{2}\right)$

$r$ radial distance from center line $(\mathrm{m})$

$R$ : width of packed bed $(\mathrm{m})$

$R_{g}$ : gas contant $\left(\mathrm{kg} \cdot \mathrm{m} / \mathrm{kgmol} \cdot{ }^{\circ} \mathrm{K}\right)$

$s$ : standard deviation concerning total pressure drop along each streamline (-)

$t$ : time (hr)

T: absolute temperature of gas $\left({ }^{\circ} \mathrm{K}\right)$

$\vec{v}$ : velocity vector of gas $(\mathrm{m} / \mathrm{hr})$

$u, v$ : lateral and longitudinal components of velocity vector, respectively $(\mathrm{m} / \mathrm{hr})$

$V:$ volume of layer of solid particles $\left(\mathrm{m}^{3}\right.$ (bed))

$w$ : thickness of packed bed (m)

$\alpha$ : apparent angle of repose of layer of solid particles (-)

\lrcorner : vertical depth of layer of solid particles $(\mathrm{m})$

$\varepsilon$ : voidage $(-)$

$\zeta:$ dimensionless lateral distance, $=r / R_{0} \quad(-)$

$\mu$ : viscosity of gas $(\mathrm{kg} / \mathrm{m} \cdot \mathrm{hr})$

$\xi:$ dimensionless height, $=l / R_{0} \quad(-)$

$\rho:$ density of gas $\left(\mathrm{kg} / \mathrm{m}^{3}\right)$

$\phi:$ shape factor (-)

$\psi$ : stream function $(\mathrm{kg} / \mathrm{m} \cdot \mathrm{hr})$

$\Psi:$ drag coefficient in Ergun's equation (-)

$\omega$ : relaxation parameter (-)

\section{Subscripts}

0: bottom of bed

1: coke layer

2: ore layer

in: inlet

out: outlet 


\section{REFERENCES}

1) K. Polthier: Arch. Eisenhüttenw., 37 (1966), 453.

2) J. Radestock and R. Jeschar: Stahl u. Eisen, 90 (1970), 1249.

3) V.S. Shvydkıi, Ya. M. Gordon, Yu. G. Yaroshenko and V. B. Shcherbatskii: Izv. VUZov, Cher. Met., (1974), No. 8, 137.

4) V. Stanek and J. Szekely: Can. J. Chem. Eng., 50 (1972), 9.

5) K. Araki, A. Moriyama and K. Nishio: Tetsu-to-Hagané, 60
(1974), 2085.

6) M. Kuwabara and I. Muchi: Tetsu-to-Hagané, 61 (1975), 787; Trans. ISIJ, 17 (1977), 321

7) S. Ergun: Cem. Eng. Progr., 48 (1952), 89.

8) K. Kanbara, T. Hagihara, M. Sasaki, Y. Okuno, T. Katayama and K. Yoshizawa: Tetsu-to-Hagané, 59 (1973), A77.

9) K. Ono, Y. Hida, A. Shigemi and K. Kodama: Tetsu-toHagané, 61 (1975), 777. 